\title{
Intestinal HIF-1a Deletion Exacerbates Alcoholic Liver Disease through Inducing Intestinal Dysbiosis and Barrier Dysfunction
}

\author{
Tuo Shao ${ }^{1,2,3}$, Cuiqing Zhao ${ }^{2,4}$, Fengyuan $\mathbf{L i}^{1,2}$, Zelin Gu ${ }^{2,5}$, Limimg Liu ${ }^{2,4}$, Lihua Zhang ${ }^{2}$, \\ Yuhua Wang ${ }^{5}$, Liqing He ${ }^{6}$, Yunhuan $\mathrm{Liu}^{2}$, Qi Liü ${ }^{2,7}$, Yiping Chen ${ }^{7}$, Hridgandh Donde ${ }^{2}$, Rui \\ Wang $^{3}$, Venkatakrishna R. Jala ${ }^{8}$, Shirish Barve ${ }^{1,2}$, Shao-Yu Chen ${ }^{1}$, Xiang Zhang ${ }^{1,6}$,

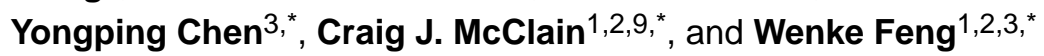 \\ ${ }^{1}$ Department of Pharmacology and Toxicology, University of Louisville, Louisville, KY, USA \\ ${ }^{2}$ Department of Medicine, University of Louisville, Louisville, KY, USA \\ ${ }^{3}$ First Affiliated Hospital, Wenzhou Medical Univesity, Wenzhou, Zhejiang, China \\ ${ }^{4}$ Institute of Virology, Wenzhou University, Wenzhou, Zhejiang, China \\ ${ }^{5}$ College of Food Science and Technology, Jilin Agricultural University, Changchun, Jilin, China \\ ${ }^{6}$ Department of Chemistry, University of Louisville, Louisville, KY, USA \\ ${ }^{7}$ Second Affiliated Hospital, Wenzhou Medical Univesity, Wenzhou, Zhejiang, China \\ ${ }^{8}$ Department of Microbiology and Immunology, University of Louisville, Louisville, KY, USA \\ ${ }^{9}$ Robley Rex Louisville VAMC, Louisville, KY, USA
}

\begin{abstract}
Objectives: Alcoholic liver disease (ALD) is characterized by gut dysbiosis and increased gut permeability. Hypoxia Induced Factor 1a (HIF-1a) has been implicated in transcriptional regulation of intestinal barrier integrity and inflammation. We aimed to test the hypothesis that HIF-1a plays a critical role in gut microbiota homeostasis and the maintenance of intestinal barrier integrity in a mouse model of ALD.

Methods: Wide type (WT) and intestinal epithelial-specific HIF-1a knockout mice (IEhif-1a ${ }^{-/-}$) were pair-fed modified Lieber-DeCarli liquid diet containing 5\% (w/v) alcohol or isocaloric maltose dextrin for 24 days. Serum levels of ALT and endotoxin were determined. Fecal microbiota were assessed. Liver steatosis and injury, and intestinal barrier integrity were evaluated.
\end{abstract}

\footnotetext{
*Corresponding authors: Department of Medicine, University of Louisville, 505 S. Hancock Street CTR517, Louisville, KY, United State, 40202. Tel.: +1 502852 2912; fax: +1 502852 8927; wenke.feng@ louisville.edu, or 13505777281@ 163.com or craig.mcclain@louisville.edu.

Authors Contributions: T.S. performed experiments and drafted manuscript, Z.G., F.L., C.Z., L.L., L.Z., Y.L., Q.L., L.H. and V. J. performed experiments, H.D. and S.B. provided some samples. Y.C., C.M., Y.C., S.B., S-Y.C., X. Z. and R.W. involved in concenptional discussion, and W.F. and C.M. conceived project, designed the study and wrote the manuscript.

Conflicts of Interest: The authors declare no conflict of interest.

Publisher's Disclaimer: This is a PDF file of an unedited manuscript that has been accepted for publication. As a service to our customers we are providing this early version of the manuscript. The manuscript will undergo copyediting, typesetting, and review of the resulting proof before it is published in its final citable form. Please note that during the production process errors may be discovered which could affect the content, and all legal disclaimers that apply to the journal pertain.
} 
Results: Alcohol feeding increased serum levels of ALT and LPS, hepatic triglyceride concentration, and liver injury in the WT mice. These deleterious effects were exaggerated in IEhif-1 $a^{-/-}$mice. Alcohol exposure resulted in greater reduction of the expression of intestinal epithelial tight junction proteins, claudin-1 and occludin, in IEhif-1 $a^{-/-}$mice. In addition, cathelicidin-related antimicrobial peptide (CRAMP) and intestinal trefoil factor (ITF) were further decreased by alcohol in IEhif- $1 a^{-/-}$mice. Metagenomic analysis showed an increased gut dysbiosis with a significantly decreased firmicutes/bacteroidetes ratio in IEhif- $1 \mathrm{a}^{-/-}$mice compared to the WT mice exposed to alcohol. An increased abundance of Akkermansia and a decreased level of Lactobacillus in IEhif- $1 a^{-/}$mice were observed. Non-absorbable antibiotics treatment reversed the liver steatosis in both WT and IEhif-1 $1 a^{-/}$mice.

Conclusion: Intestinal HIF-1a is essential for the adaptation response to alcohol exposureinduced changes in intestinal microbiota and barrier function associated with elevated endotoxemia and hepatic steatosis and injury.

\section{Graphical Abstract}
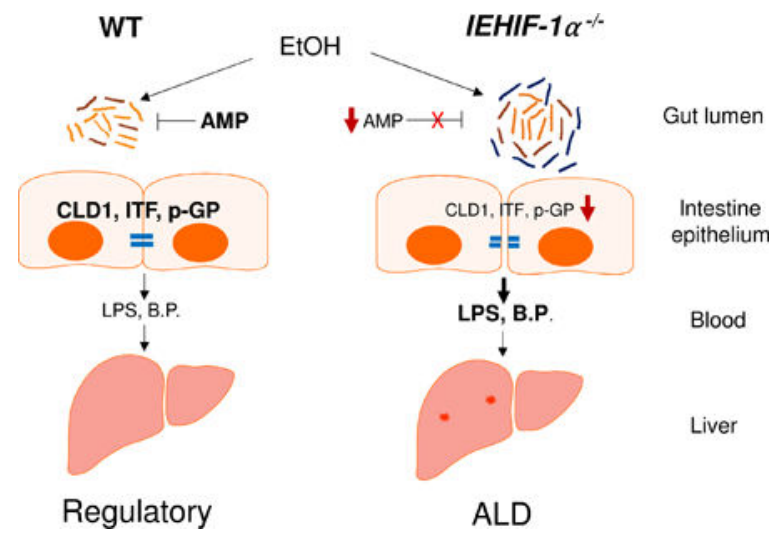

A schematic diagram illustrating the proposed mechanism by which intestinal HIF-1a depletion exacerbates alcoholic liver disease. AMP: antimicrobial peptide, CLD1: claudin-1, ITF: intestinal trefoil factor, p-GP: p-glycoprotein, B.P.: bacterial products

\section{Lay summary}

Alcohol consumption alters gut microbiota and multiple intestinal barrier protecting factors that are regulated by intestinal hypoxia inducible factor $1 a$ (HIF-1a). Absence of intestinal HIF-1a exacerbates gut leakiness leading to an increased translocation of bacteria and bacterial products into liver, and consequently causes alcoholic liver disease (ALD). Intestinal specific upregulation of HIF-1a could be developed as a novel approach for the treatment of ALD

\section{Keywords}

Hypoxia Inducible Factor-1a; Alcoholic Liver Disease; Gut Microbiota; Intestinal Barrier; GutLiver axis 


\section{INTRODUCTION}

Alcoholic liver disease (ALD) ranges from hepatic steatosis to steatohepatitis, cirrhosis, and, potentially, hepatocellular carcinoma [1]. While alcohol induces deleterious effects in the liver, it also disrupts the gut microbiota homeostasis and intestinal epithelial integrity resulting in an increased permeability, bacterial translocation and release of bacteria-derived endotoxin into circulation [2-4]. Clinical and experimental studies have demonstrated that serum levels of lipopolysaccharide (LPS) are indeed increased in alcoholic subjects [5, 6]. LPS binding to Toll-like receptors (TLRs) on the surface of Kupffer cells leads to an elevated pro-inflammatory cytokine production, which, in turn, damages hepatocyte function $[7,8]$.

Intestinal barrier function is key to preventing the increased bacterial translocation by alcohol $[5,9,10]$. Goblet cells in the intestinal epithelium produce protective trefoil factors and mucins, which are abundantly core glycosylated and either localized to the cell membrane or secreted into the lumen to form the mucus layer [11, 12]. The mucus is the first barrier that intestinal bacteria meet, and pathogens must penetrate it to reach the epithelial cells [13, 14] [15]. Intestinal Paneth cells produce antimicrobial peptides that are retained in the mucus layer, thereby concentrating their bactericidal activity close to the epithelium. Together, Paneth and Goblet cells prevent bacterial invasion to some extent, and thereby play a prominent role in the innate immune surveillance of the gut [12]. The paracellular space in the intestinal epithelium is sealed by tight junctions (TJ), which regulate the flow of small molecules through the composition of claudins and other proteins in the junctional complex [16]. IgA production and other intestinal immune-regulations provide a protection against bacterial transcytosis [17]. Recent studies demonstrate that targeting intestinal barrier function may be an effective strategy in the prevention/treatment of ALD [18].

The intestines are supported by an extensive underlying vasculature, and are therefore susceptible to conditions related diminished blood flow and concomitant tissue hypoxia [19, 20]. It is now clear that responses to hypoxia include transcriptionally regulated gene expression coordinated by the transcription factor hypoxia-inducible factor (HIF) [21]. HIF- $1 a$ and HIF-2 $a$ have been implicated in transcriptional regulation of anti-inflammatory or tissue-protective-signaling pathways [22-24]. A number of barrier-protective genes are critically regulated by HIF-1a, including intestinal trefoil factor (ITF), CD73, Pglycoprotein (P-gp), cathelicidin, claudin-1, muc3 and $\beta$-defensin-1(DEFB1) [19, 25-30]. While hypoxia is an important factor for HIF-1a protein stabilization, there are also a number of factors other than hypoxia, such as LPS, that regulate HIF-1a expression and accumulation [31]. A recent study demonstrates that long time exposure to LPS impairs HIF-1a induction, which reduces cell viability and function under hypoxia conditions [32].

Disruption of epithelial HIF-1a resulted in an increased epithelial permeability, as shown in murine colitis models [33-36]. Our previous study showed that long-term exposure to alcohol decreased epithelial HIF-2a protein expression in mice [37]. We also demonstrated that knockdown of $\mathrm{HIF}^{1} / 2 \mathrm{a}$ resulted in an increased permeability and a decreased transepithelial electrical resistance in Caco-2 cells [37, 38]. 
In the present study, we discovered that intestinal epithelial specific HIF-1a knockout (IEhifl $a^{-/-}$) worsens alcohol-induced liver injury. We showed that alcohol exposure in IEhif $1 a^{-/}$mice resulted in a more severe dysbiosis, increased hepatic bacterial products, and liver injury. IEhifl $1 a^{-/}$mice had decreased levels of antimicrobial peptide in the intestine and an increased intestinal inflammation. Probiotic administration decreased alcohol-induced liver injury in wild type (WT) mice but not in IEhifl $1 a^{-/}$mice. Sterilizing the gut using non-absorbable antibiotics attenuated liver-injury in IEhifla ${ }^{-/-}$mice. Our results suggest that targeting intestinal HIF-1a may be a strategy for preventing ALD.

\section{MATERIALS AND METHODS}

\section{Animals}

All animal protocols were approved by the Institutional Animal Care and Use Committee of the University of Louisville.

Other methods and detailed information ragerdaing animal treatments are provided in the Supplementary materials.

For further details regarding the materials used in this study, please refer to the CTAT table in the Suppelementary Materials.

\section{RESULTS}

\section{IEhif1 $^{-/-}$Mice Have Exacerbated Alcohol-Induced Hepatic Steatosis}

We examined the intestinal expression of HIF-1a in mice treated with alcohol at different time points. 12- and 24-day alcohol exposure increased, while 48-day exposure decreased, intestinal HIF-1a mRNA expression (Fig. 1A). We hypothesized that intestinal HIF-1a upregulation is an adaptive response to alcohol exposure, and prolonged alcohol treatment damages this adaptive mechanism. Depleting HIF-1a may disrupt this HIF-1a-mediated intestinal homeostasis and result in liver injury. Therefore, we used intestinal epithelial specific HIF-1a knockout (IEhifla ${ }^{-/}$) mice to examine the intestine and liver pathophysiology in response to 24-day alcohol treatment. mRNA levels of HIF-1a in IEhifl $\mathrm{a}^{-/}$mice decreased significantly in the intestine, but not in the liver (Supplementary. Fig. 1A). To further confirm the deletion effectiveness, the chemical hypoxia inducer, $\mathrm{CoCl}_{2}$, was administered to mice via intraperitoneal injection, and liver and intestine HIF-1a protein was immunoblotted. $\mathrm{CoCl}_{2}$ induced a prominent increase in HIF-1a protein in the liver, but the changes were minimal in intestine (Supplementary. Fig.1B). The residual expression of HIF-1a in the intestinal tissue of IEhifla ${ }^{-/}$mice was due to the expression of HIF-1a in other types of intestinal cells, such as dendritic cells [39], intestinal macrophages [40] and T cells [41, 42].

Chronic alcohol feeding decreased survival rate of IEhifla $a^{-/-}$mice compared to WT mice (Fig. 1B). IEhifla $a^{-/}$mice had pronounced increase in hepatic steatosis, compared to the WT mice after alcohol treatment (Fig. 1C and 1D). Liver to body weight ratio was not changed in WT mice, but significantly increased in IEhifla ${ }^{-/-}$mice (Fig. 1E). Liver triglyceride level was increased in WT mice, but markedly increased in the IEhifla ${ }^{-/-}$mice 
(Fig. 1F). Alcohol feeding significantly increased hepatic SREBP-1 mRNA level in both WT and IEhifl $1 a^{-/}$mice, but the effect was much greater in IEhifl $a^{-/}$mice, indicating an increased lipogenesis in IEhifla ${ }^{-/-}$mice upon alcohol exposure (Fig.1G).

Fasting serum glucose level was slightly decreased by alcohol feeding in WT mice. However, the KO mice had significantly higher glucose levels (Supplementary Fig. 2A). Lipid panel measurements showed that levels of serum triglyceride, total cholesterol, LDL, HDL and VLDL were slightly lower in WT mice, but significantly increased in IEhifla ${ }^{-/-}$ mice (Supplementary. Fig. 2B-2F).

\section{Alcohol Increases Hepatic Inflammatory Response in IEhif1 ${ }^{-/}$mice}

Alcohol feeding increased hepatic inflammation in IEhifla ${ }^{-/-}$mice. As shown in Fig. 2A, IEhifl $\mathrm{a}^{-/}$mice had an increased infiltration of cytotoxic neutrophils (chloracetate esterase CAE staining) in the liver. Additionally, immunohistochemical staining using F4/80 revealed more macrophages in the livers of IEhifla ${ }^{-/}$mice (Fig. 2B). More intense neutrophil infiltration and macrophage activation in livers of IEhifla ${ }^{-/-}$mice were associated with elevated gene expression of the pro-inflammatory factors, TNF- $a$ and IL-6, and the chemokine MCP-1 (Fig. 2C, 2D and 2E). Hepatic TLR4 protein increased in IEhifla ${ }^{-/}$ mice after alcohol exposure (Supplementary Fig. 3). Serum level of ALT was increased in both WT and IEhifla ${ }^{-/-}$mice (Fig. 2F). Taken together, these results demonstrate that IEhif $1 a^{-/-}$mice have enhanced hepatic steatosis and inflammation upon chronic alcohol exposure.

\section{Alcohol Increases Hepatic Fibrosis in IEhif1 $a^{-/-}$mice}

IEhifla ${ }^{-/-}$mice had a significant increase in Sirius Red positive area compared to WT mice (Supplementary Fig. 4A). The gene expression of fibrogenic factors, collagen $\mathrm{a} 1$ and aSMA were marginally increased in the WT mice, but were significantly increased in IEhifl $a^{-/-}$mice (Supplementary Fig. 4B and 4C). This suggests that intestinal epithelial HIF-1a depletion exacerbates hepatic fibrosis following alcohol exposure.

\section{IEhif1a ${ }^{-/-}$Mice Exhibit Gut Dysbiosis}

Previous studies showed that ALD is associated with gut dysbiosis and endotoxin release. We next determined whether gut bacterial homeostasis and the translocation of bacterial products are affected by the absence of intestinal Hifla. As shown in Fig. 3A, total bacterial load was unchanged in the WT mice, but significantly increased in the IEhifla ${ }^{-/-}$mice after 24 days of chronic alcohol feeding, indicating a bacterial overgrowth. Metagenomic analysis showed that alcohol feeding markedly increased phylum levels of Bacteroidetes and decreased Firmicutes in IEhif1 $a^{-/}$mice (Fig. 3B). The ratio of Firmucutes/Bacteriodetes was not altered by alcohol exposure in the WT mice, but was significantly decreased in the KO mice (Fig. 3C). Further analysis showed that Akkermansia spp. was increased in IEhifl $a^{-/-}$mice but not in the WT mice (Fig. 3D). Interestingly, Lactobacillus spp. was decreased by alcohol in WT mice, and was almost undetectable in IEhifla ${ }^{-1-}$ mice (Fig. $3 \mathrm{E})$. These data indicated that IEhif $1 a^{-/-}$mice had an exacerbated gut dysbiosis following 24-day alcohol exposure. 
Gut bacterial homeostasis is regulated by multiple anti-microbial peptides, which are secreted by intestinal epithelium. Alcohol exposure decreased the numbers of Goblet cells in the mucosal tissues of both WT and IEhifla ${ }^{-/-}$mice, but the effect was greater in IEhifla ${ }^{-}$mice (Supplementary Fig. 5). $\beta$-defensins are the dominant class of antimicrobial peptides secreted by the epithelium. The gene expression of $\beta$-defensin- 1 and -2 was increased by alcohol exposure in the ileal tissue of WT mice, while it remained unchanged in the IEhifla ${ }^{-/}$mice (Fig. 3F and 3G). Cathelicidin related antimicrobial peptide (CRAMP) is the only member of cathelicidin anti-microbial peptide family in mice. Alcohol exposure significantly reduced CRAMP expression in both WT and IEhifla ${ }^{-/-}$mice (Fig. $3 \mathrm{H})$.

\section{Induction of HIF in the Intestinal Epithelial Cells Inhibits Mouse Fecal Bacterial Growth}

The role of intestinal HIF-1a in gut bacterial growth was further analyzed in an in vitro assay. Human T84 intestinal epithelial cells were incubated with DMOG (Dimethyloxaloylglycine), a HIF-1a activator. The conditioned medium was added to the mouse fecal bacterial culture, and bacterial growth rate was determined. Neither DMOG itself nor control medium changed the fecal bacterial growth over the period of 6 hours. DMOG-conditioned medium, presuming containing stimulated antimicrobial peptides, significantly inhibited bacterial growth (Fig. 4A). However, this inhibitory effect was abolished when using the medium from the HIF-1a dominant negative (HIF-1aDN) plasmid transfected cells, indicating HIF-1a-mediated secreted factors from epithelial cells likely possess anti-microbial capacity. To further confirm whether HIF-1a activation can increase anti-microbial capabilities, we determined anti-microbial peptide expression. The gene expression of human $\beta$-defensin-1, -2 (hBD-1, -2) and human cathelicidin related antimicrobial peptide (LL-37) was increased significantly by DMOG in T84 cells (Fig. 4BD). Protein levels of LL-37 in the DMOG-conditioned medium were increased, but were reduced in the medium of HIF1a-DN transfected cells (Fig. 4E). These results indicate that HIF-1a activation increases antimicrobial peptide expression in epithelial cells.

\section{HIF1a-deficiency Induces Intestinal Barrier Dysfunction After Chronic Alcohol Exposure}

Increased bacterial product translocation indicates intestinal barrier dysfunction. Immunofluorescent staining showed a decreased expression of TJ proteins, occludin and claudin-1, in the ileal tissues of WT mice after alcohol exposure. Importantly, these decreases were more severe in IEhifla ${ }^{-/-}$mice (Fig. 5A and 5B). The decreased TJ protein expression was associated with the disruption of intestinal protective factors, such as ITF and P-gp. The protein level of ITF was significantly lower in the IEhifl $a^{-/}$mice compared to the WT mice (Fig. 5C). Furthermore, P-gp plays an important role in overall intestinal barrier integrity, and it was upregulated by alcohol in WT mice. This adaptive increase was, however, eliminated in the IEhif1 $a^{-/}$mice (Fig. 5D).

\section{Alcohol Increases Intestinal Inflammatory Response in IEhif1 $a^{-/-}$Mice}

Histologic examination revealed a prominent inflammatory response in the ileum of IEhifla -/ mice exposed to alcohol (Fig. 6A). Alcohol feeding significantly increased macrophagelike appearance in Peyer's patches (PPs), a major component of the gut-associated lymphoid tissue, serving as important antigen entry sites in mucosal immunity. The PP domes were 
irregular, and the surrounding villi were sparse and atrophic (Fig. 6A), indicating an increased intestinal inflammatory response in the small intestines of IEhifla ${ }^{-/-}$mice exposed to alcohol. We further analyzed alcohol-induced intestinal inflammation. Immunofluorescent analysis revealed an increased number of cells expressing F4/80 in alcohol-treated-IEhif $1 a^{-/-}$mice compared with WT mice (Fig. 6B). Intestinal inflammation was further confirmed by the increased mRNA levels of proinflammatory cytokines, IL-6, IL-1 $\beta$ and TNF-a (Fig. 6C-6E).

\section{Alcohol Increases Intestinal Permeability}

Increased intestinal barrier damage and inflammation leads to higher intestinal permeability and induces greater bacteria translocation. To further determine the role of epithelial HIF-1a in alcohol-induced translocation of bacteria/bacterial products to the liver, we analyzed circulating LPS concentration and Escherichia coli (E. coli) protein in the liver. Serum LPS level was slightly elevated by alcohol feeding in the WT mice, but significantly increased in IEhifla ${ }^{-/-}$mice (Supplementary Fig. 6A). Furthermore, hepatic content of E. coli protein was increased in WT mice following alcohol exposure, and this increase was more pronounced in IEhifla ${ }^{-/}$mice, as evaluated by immunofluorescent staining of hepatic tissues (Supplementary Fig. 6B).

\section{Antibiotic Sterilization of the Gut Reduces Alcohol-Induced Liver Fat Accumulation and Injury}

To further elucidate the role of gut bacteria in alcohol-induced liver steatosis and injury in IEhif1 $a^{-/-}$mice, non-absorbable antibiotics (ABx; Polymyxin B and Neomycin) were administered to the mice exposed to alcohol for 12 days. $\mathrm{ABx}$ treatment decreased hepatic fat accumulation (Fig. 7A), liver triglyceride levels (Fig. 7B) and serum ALT levels (Fig. 7C) in IEhifla $a^{-/}$mice. These results support the concept that gut dysbiosis is critical in alcohol-induced hepatic steatosis and injury in the IEhifla $a^{-/}$mice.

\section{HIF-1a is Required for the Protective Effect of Lactobacillus rhamnosus GG in ALD}

Our previous studies have shown that the probiotic, LGG, protects against ALD by increasing intestinal HIF signaling and modifying gut bacterial homeostasis [37, 38]. In this study, we tested whether HIF-1a is required for the protective effects of LGG. IEhifla ${ }^{-/-}$ and WT mice were fed 5\% (w/v) alcohol for 10 days and a single dose of alcohol was gavaged in the morning of last day (NIAAA model) [43]. LGG was administered by gavage at a dose of $10^{9} \mathrm{CFU} /$ day along with alcohol exposure. Consistent with our previous study, LGG administration significantly reduced hepatic fat accumulation in WT mice. However, this protective effect was diminished in IEhifl $a^{-/}$mice (Fig. 8A and 8B). Plasma ALT and AST level showed a modest improvement with LGG treatment in WT mice, but not in IEhifl $a^{-/}$mice (Fig. 8C and 8D). In addition, LGG supplementation increased fecal butyrate concentration in WT, but not in IEhifla ${ }^{-/-}$mice (Fig. 8E). Intestinal barrier protective gene expression of claudin-1 and P-gp was upregulated in the WT mice, but not in the IEhif $1 a^{-/-}$mice following LGG treatment. These results suggest that HIF-1a is required for the beneficial effects of LGG in the reduction of steatosis in ALD. 


\section{DISCUSSION}

The gut-liver axis plays an important role in the pathogenesis of chronic liver disease. Intestinal dysbiosis and the disruption of the intestinal barrier integrity by alcohol are prominent features of ALD. Previous studies demonstrated that alcohol exposure-associated changes in gut metabolites and antimicrobial peptide production contribute to ALD [5, 9, 18]. The mechanisms by which alcohol disrupts the antimicrobial peptide response leading to dysbiosis and intestinal barrier dysfunction are not fully understood. In this study, we showed that deletion of intestinal HIF-1a increased gut dysbiosis and intestinal integrity, resulting in a exacerbated liver steatosis, inflammation, fibrosis and injury.

Our previous studies have shown that long-term ( 8 weeks) alcohol exposure decreased HIF-2a protein levels in mouse intestine and increased gut leakiness and liver injury [37]. A recent study demonstrated that high fat diet treatment promotes HIF- $2 a$ activation but not HIF-1a signaling in the intestine, which contributes to hepatic steatosis in obesity through ceramide metabolism mediated by Neu3 regulation [44]. However, the role of intestinal HIF-1a in ALD has not been evaluated. The increase in HIF-1a signaling resulting from the relatively short alcohol exposure (12 and 24 days) likely represents an adaptive upregulation. However, this adaptive response is decreased by prolonged alcohol exposure. How intestinal HIF-1a is regulated in alcoholic pateints is not yet known, but the expression of intestinal claudin-1, an HIF-1a target, was reduced in alcoholic cirrhrosis patients [45].

Pathogenic bacteria overgrowth and reduction of beneficial bacteria constitute gut dysbiosis in ALD. The gut microbiota homeostasis is controlled by bacteria-bacteria and bacteria-host interactions under both healthy and diseased conditions. Alcohol exposure disrupts microbiota homeostasis. However, the gut mucosal response has adaptive mechanisms to restore homeostasis by increasing antimicrobial substances. We postulate that epithelial HIF-1a represents such an adaptive mechanism. 24-day alcohol exposure modestly changed gut microbiota homeostasis in WT mice. However, depletion of intestinal epithelial HIF-1a resulted in a more severe dysbiosis. The IEhifl $1 a^{-/}$mice had reduced fecal Firmicutes and increased Bacteroidetes. Such a change has been shown in a mouse model of ALD using intragastric feeding [46]. At the bacterial species level, Akkermansia (Akk), gram-negative anaerobic bacteria, were not affected by alcohol in WT mice, but were increased in the IEhifl ${ }^{-/-}$mice. The role of Akk in ALD remains elusive. Supplementation with Akk showed a protective effect in ALD [46], while endogenous increase of Akk may impact the intestinal innate immune system and facilitate bacterial translocation by degrading the intestinal mucus [47-49]. The causal relationship between an intestinal increase in Akk and intestinal integrity needs further study. Interestingly, Lactobacillus bacteria were markedly depleted by 24-day alcohol exposure in WT mice, and this decrease was greater in IEhifla ${ }^{-}-$mice. A recent study demonstrated that Lactobacillus utilized saturated long-chain fatty acids, which were beneficial in ALD treatment [50]. A short-chain fatty acid, butyrate, plays an essential role in epithelial cell nutrition and intestinal physiology. Butyrate regulates epigenetic processes through HDAC inhibition. Intestinal consumption of butyrate causes local hypoxia leading to increased HIF-1a expression [51]. Although Lactobacillus itself does not produce butyrate, studies have shown that Lactobacillus promotes eubiosis, thereby resulting increased butyrate production. Butyrate-producing bacteria are mainly in the 
phylum Firmucutes, which was decreased by alcohol in our study. Furthermore, the decrease of Lactobacillus in ALD is consistent with the beneficial effect of LGG supplementation on ALD as shown by several groups [37, 38, 52-54].

HIF-1a is a master transcription factor regulating a variety of genes in the intestine. Antimicrobial peptides, $\beta$-defensin 1 and CRAMP, are regulated by HIF-1a [27, 30]. Paneth cell-derived $\beta$-defensins and epithelial cell- and microphage-derived CRAMP regulate gut microbiota homeostasis. Our results showed that $\beta$-defensins were adaptively increased and CRAMP was decreased by alcohol exposure in WT mice. However, depleting HIF-1a eliminated $\beta$-defensin upregulation and further decreased CRAMP. This, at least partially, explains why IEhifla ${ }^{-/-}$mice developed dysbiosis following alcohol exposure. In contrast, activation of HIF-1a in epithelial T84 cells inhibited bacterial growth and increased antimicrobial gene expression. In vivo activation of HIF-1a by DMOG demonstrated a partial prevention of ALD (Supplementary Fig. 7A and 7B). However, this observation is not conclusive because DOMG treatment is not intestine specific. While intestinal HIF-1a might be beneficial in ALD, hepatic activation of HIF-1a showed contradictory results [55, 56]. The causative role of gut dysbiosis in IEhifla ${ }^{-/-}$mice in ALD was further demonstrated by sterilization of the gut using non-absorbable antibiotics.

IEhifla ${ }^{-/-}$mice had an increase in intestinal permeability in ALD, which has been shown in inflammatory bowel disease [28, 33]. Subsequently, bacteria and bacterial products translocate to the systemic circulation and liver. Indeed, IEhifla ${ }^{-/}$mice displayed a higher LPS level in the serum and increased E. coli protein in the liver after alcohol feeding. LPS binds to TLR4 on hepatocytes and Kupffer cells resulting in a hepatic fat accumulation and inflammation, which is an established and well-studied mechanism in chronic ALD.

In addition to the effect of HIF-1a on antimicrobial peptide regulation, HIF-1a also regulates several critical factors that are important in intestinal barrier integrity. P-gp functions as a pump by expelling translocated xenobiotics and bacterial toxins from the enterocytes into the intestinal lumen [26]. ITF is a mucus modifier that helps to maintain a dynamic mucus layer [19]. Claudin-1 is a major tight junction protein in the intestinal epithelium [28]. Reduction in ITF, claudin-1 and P-gp in IEhifla ${ }^{-/-}$mice following alcohol exposure destabilizes gut barrier function, resulting in an elevated LPS concentration in serum and E. coli protein in the liver. In addition, alcohol exposure increases intestinal Cyp2E1 expression [57], which is responsible for the production of ROS. Furthermore, alcohol feeding significantly increased intestinal inflammation in IEhifla ${ }^{-/-}$mice. Taken together, these multiple mechanisms contribute to the exacerbated gut barrier dysfunction in IEhifla $^{-/-}$mice in ALD.

Our previous studies demonstrated that the probiotic, LGG, is beneficial in preventing/ treating of ALD in mice. However, the beneficial effects were diminished when HIF-1a was deleted in the intestine. LGG is able to increase the lumenal concentrations of long-chain fatty acids and SCFAs in mice exposed to alcohol. Lactobacilli, in turn, metabolize saturated fatty acids to promote their growth. On the other hand, supplementation with tributyrin, a prodrug of butyrate, protects from intestinal tight junction disruption by alcohol [45].

Currently, it is still unclear as to how butyrate protects tight junctions. However, butyrate 
metabolism in the intestine consumes oxygen and stabilizes HIF-1a [51], which protects the intestinal barrier. Thus, it is likely that LGG promotes butyrate-producing bacteria growth, and the resulting butyrate production increases HIF-1a stabilization and protects the intestinal barrier. Intestinal HIF-1a depletion abolishes this protective effect of LGG against alcohol-induced intestinal barrier disruption.

In conclusion, our findings demonstrate that intestinal epithelial HIF- $1 \alpha$ is critically involved in the intestinal pathogenesis of alcohol-induced barrier disruption and subsequent liver injury. HIF-1a activation regulates gut bacterial homeostasis though increasing antimicrobial peptides and stabilizes barrier function via upregulation of P-gp and tight junction proteins. Because systemic activation of HIF-1a may have deleterious effects in the liver, our study suggests that dietary approaches and pharmacological agents that specifically activate intestinal HIF-1a could be developed to prevent/treat ALD.

\section{Supplementary Material}

Refer to Web version on PubMed Central for supplementary material.

\section{ACKNOWLEDGMENTS}

We thank Marion McClain for the manuscript proofreading.

Financial support: The work presented in this study was supported by NIH grants R21 AA002416, R01AA023190, R01AA023681, R01AA020265, R01AA021434, U01AA022489, U01AA021901, P50AA024337, P20GM113226, the VA (1I01BX002996-01A2) and grants from Chinese National Science Foundation (81370481, $81770585,81600466,81570514)$.

\section{REFERENCES}

[1]. Gao B, Bataller R. Alcoholic Liver Disease: Pathogenesis and New Therapeutic Targets. Gastroenterology 2011;141:1572-1585. [PubMed: 21920463]

[2]. Adachi Y, Moore LE, Bradford BU, Gao W, Thurman RG. Antibiotics prevent liver injury in rats following long-term exposure to ethanol. Gastroenterology 1995;108:218-224. [PubMed: 7806045]

[3]. Parlesak A, Schafer C, Schutz T, Bode JC, Bode C. Increased intestinal permeability to macromolecules and endotoxemia in patients with chronic alcohol abuse in different stages of alcohol-induced liver disease. J Hepatol 2000;32:742-747. [PubMed: 10845660]

[4]. Chen P, Miyamoto Y, Mazagova M, Lee KC, Eckmann L, Schnabl B. Microbiota Protects Mice Against Acute Alcohol-Induced Liver Injury. Alcohol Clin Exp Res 2015;39:2313-2323. [PubMed: 26556636]

[5]. Szabo G, Bala S. Alcoholic liver disease and the gut-liver axis. World J Gastroenterol 2010;16:1321-1329. [PubMed: 20238398]

[6]. Kirpich IA, McClain CJ, Vatsalya V, Schwandt M, Phillips M, Falkner KC, et al. Liver Injury and Endotoxemia in Male and Female Alcohol-Dependent Individuals Admitted to an Alcohol Treatment Program. Alcohol Clin Exp Res 2017;41:747-757. [PubMed: 28166367]

[7]. Uesugi T, Froh M, Arteel GE, Bradford BU, Thurman RG. Toll-like receptor 4 is involved in the mechanism of early alcohol-induced liver injury in mice. Hepatology 2001;34:101-108. [PubMed: 11431739]

[8]. Petrasek J, Mandrekar P, Szabo G. Toll-like receptors in the pathogenesis of alcoholic liver disease. Gastroenterology research and practice 2010;2010. 
[9]. Keshavarzian A, Holmes EW, Patel M, Iber F, Fields JZ, Pethkar S. Leaky gut in alcoholic cirrhosis: a possible mechanism for alcohol-induced liver damage. The American journal of gastroenterology 1999;94:200-207. [PubMed: 9934756]

[10]. Wang Y, Tong J, Chang B, Wang B, Zhang D. Effects of alcohol on intestinal epithelial barrier permeability and expression of tight junction-associated proteins. Molecular medicine reports 2014;9:2352-2356. [PubMed: 24718485]

[11]. Cheng H, Leblond CP. Origin, differentiation and renewal of the four main epithelial cell types in the mouse small intestine. V. Unitarian Theory of the origin of the four epithelial cell types. The American journal of anatomy 1974;141:537-561. [PubMed: 4440635]

[12]. Zhao F, Edwards R, Dizon D, Afrasiabi K, Mastroianni JR, Geyfman M, et al. Disruption of Paneth and goblet cell homeostasis and increased endoplasmic reticulum stress in Agr2-/- mice. Dev Biol 2010;338:270-279. [PubMed: 20025862]

[13]. Johansson ME, Phillipson M, Petersson J, Velcich A, Holm L, Hansson GC. The inner of the two Muc2 mucin-dependent mucus layers in colon is devoid of bacteria. Proc Natl Acad Sci U S A 2008;105:15064-15069. [PubMed: 18806221]

[14]. Van der Sluis M, De Koning BA, De Bruijn AC, Velcich A, Meijerink JP, Van Goudoever JB, et al. Muc2-deficient mice spontaneously develop colitis, indicating that MUC2 is critical for colonic protection. Gastroenterology 2006;131:117-129. [PubMed: 16831596]

[15]. Taupin D, Podolsky DK. Trefoil factors: initiators of mucosal healing. Nature Reviews Molecular Cell Biology 2003;4:721-732. [PubMed: 14506475]

[16]. Forster C Tight junctions and the modulation of barrier function in disease. Histochemistry and cell biology 2008;130:55-70. [PubMed: 18415116]

[17]. Hapfelmeier S, Lawson MA, Slack E, Kirundi JK, Stoel M, Heikenwalder M, et al. Reversible microbial colonization of germ-free mice reveals the dynamics of $\operatorname{IgA}$ immune responses. Science (New York, NY) 2010;328:1705-1709.

[18]. Szabo G Gut-liver axis in alcoholic liver disease. Gastroenterology 2015;148:30-36. [PubMed: 25447847]

[19]. Furuta GT, Turner JR, Taylor CT, Hershberg RM, Comerford K, Narravula S, et al. Hypoxiainducible factor 1-dependent induction of intestinal trefoil factor protects barrier function during hypoxia. The Journal of experimental medicine 2001;193:1027-1034. [PubMed: 11342587]

[20]. Taylor CT, Colgan SP. Therapeutic targets for hypoxia-elicited pathways. Pharmaceutical research 1999;16:1498-1505. [PubMed: 10554089]

[21]. Cramer T, Yamanishi Y, Clausen BE, Forster I, Pawlinski R, Mackman N, et al. HIF-1alpha is essential for myeloid cell-mediated inflammation. Cell 2003;112:645-657. [PubMed: 12628185]

[22]. Sitkovsky M, Lukashev D. Regulation of immune cells by local-tissue oxygen tension: HIF1 alpha and adenosine receptors. Nature reviews Immunology 2005;5:712-721.

[23]. Morote-Garcia JC, Rosenberger P, Kuhlicke J, Eltzschig HK. HIF-1-dependent repression of adenosine kinase attenuates hypoxia-induced vascular leak. Blood 2008;111:5571-5580. [PubMed: 18309031]

[24]. Glover LE, Bowers BE, Saeedi B, Ehrentraut SF, Campbell EL, Bayless AJ, et al. Control of creatine metabolism by HIF is an endogenous mechanism of barrier regulation in colitis. Proc Natl Acad Sci U S A 2013;110:19820-19825. [PubMed: 24248342]

[25]. Synnestvedt K, Furuta GT, Comerford KM, Louis N, Karhausen J, Eltzschig HK, et al. Ecto-5' nucleotidase (CD73) regulation by hypoxia-inducible factor-1 mediates permeability changes in intestinal epithelia. J Clin Invest 2002;110:993-1002. [PubMed: 12370277]

[26]. Comerford KM, Wallace TJ, Karhausen J, Louis NA, Montalto MC, Colgan SP. Hypoxiainducible factor-1-dependent regulation of the multidrug resistance (MDR1) gene. Cancer Res 2002;62:3387-3394. [PubMed: 12067980]

[27]. Fan D, Coughlin LA, Neubauer MM, Kim J, Kim MS, Zhan X, et al. Activation of HIF-1alpha and LL-37 by commensal bacteria inhibits Candida albicans colonization. Nat Med 2015;21:808814. [PubMed: 26053625]

[28]. Saeedi BJ, Kao DJ, Kitzenberg DA, Dobrinskikh E, Schwisow KD, Masterson JC, et al. HIFdependent regulation of claudin-1 is central to intestinal epithelial tight junction integrity. Mol Biol Cell 2015;26:2252-2262. [PubMed: 25904334] 
[29]. Louis NA, Hamilton KE, Canny G, Shekels LL, Ho SB, Colgan SP. Selective induction of mucin-3 by hypoxia in intestinal epithelia. J Cell Biochem 2006;99:1616-1627. [PubMed: 16823775]

[30]. Kelly CJ, Glover LE, Campbell EL, Kominsky DJ, Ehrentraut SF, Bowers BE, et al. Fundamental role for HIF-1alpha in constitutive expression of human beta defensin-1. Mucosal Immunol 2013;6:1110-1118. [PubMed: 23462909]

[31]. Siegert I, Schodel J, Nairz M, Schatz V, Dettmer K, Dick C, et al. Ferritin-Mediated Iron Sequestration Stabilizes Hypoxia-Inducible Factor-1alpha upon LPS Activation in the Presence of Ample Oxygen. Cell reports 2015;13:2048-2055. [PubMed: 26628374]

[32]. Frede S, Stockmann C, Winning S, Freitag P, Fandrey J. Hypoxia-inducible factor (HIF) 1alpha accumulation and HIF target gene expression are impaired after induction of endotoxin tolerance. J Immunol 2009;182:6470-6476. [PubMed: 19414801]

[33]. Karhausen J, Furuta GT, Tomaszewski JE, Johnson RS, Colgan SP, Haase VH. Epithelial hypoxia-inducible factor-1 is protective in murine experimental colitis. J Clin Invest 2004;114:1098-1106. [PubMed: 15489957]

[34]. Campbell EL, Bruyninckx WJ, Kelly CJ, Glover LE, McNamee EN, Bowers BE, et al. Transmigrating neutrophils shape the mucosal microenvironment through localized oxygen depletion to influence resolution of inflammation. Immunity 2014;40:66-77. [PubMed: 24412613]

[35]. Curtis VF, Ehrentraut SF, Campbell EL, Glover LE, Bayless A, Kelly CJ, et al. Stabilization of HIF through inhibition of Cullin-2 neddylation is protective in mucosal inflammatory responses. Faseb j 2015;29:208-215. [PubMed: 25326537]

[36]. Keely S, Campbell EL, Baird AW, Hansbro PM, Shalwitz RA, Kotsakis A, et al. Contribution of epithelial innate immunity to systemic protection afforded by prolyl hydroxylase inhibition in murine colitis. Mucosal Immunol 2014;7:114-123. [PubMed: 23695513]

[37]. Wang Y, Kirpich I, Liu Y, Ma Z, Barve S, McClain CJ, et al. Lactobacillus rhamnosus GG treatment potentiates intestinal hypoxia-inducible factor, promotes intestinal integrity and ameliorates alcohol-induced liver injury. Am J Pathol 2011;179:2866-2875. [PubMed: 22093263]

[38]. Wang Y, Liu Y, Sidhu A, Ma Z, McClain C, Feng W. Lactobacillus rhamnosus GG culture supernatant ameliorates acute alcohol-induced intestinal permeability and liver injury. Am $\mathrm{J}$ Physiol Gastrointest Liver Physiol 2012;303:G32-41. [PubMed: 22538402]

[39]. Fluck K, Breves G, Fandrey J, Winning S. Hypoxia-inducible factor 1 in dendritic cells is crucial for the activation of protective regulatory $\mathrm{T}$ cells in murine colitis. Mucosal Immunol 2016;9:379-390. [PubMed: 26220168]

[40]. Ortiz-Masia D, Cosin-Roger J, Calatayud S, Hernandez C, Alos R, Hinojosa J, et al. Hypoxic macrophages impair autophagy in epithelial cells through Wnt1: relevance in IBD. Mucosal Immunol 2014;7:929-938. [PubMed: 24301659]

[41]. Higashiyama M, Hokari R, Hozumi H, Kurihara C, Ueda T, Watanabe C, et al. HIF-1 in T cells ameliorated dextran sodium sulfate-induced murine colitis. J Leukoc Biol 2012;91:901-909. [PubMed: 22457366]

[42]. Colgan SP, Taylor CT. Hypoxia: an alarm signal during intestinal inflammation. Nat Rev Gastroenterol Hepatol 2010;7:281-287. [PubMed: 20368740]

[43]. Bertola A, Mathews S, Ki SH, Wang H, Gao B. Mouse model of chronic and binge ethanol feeding (the NIAAA model). Nature protocols 2013;8:627-637. [PubMed: 23449255]

[44]. Xie C, Yagai T, Luo Y, Liang X, Chen T, Wang Q, et al. Activation of intestinal hypoxiainducible factor 2alpha during obesity contributes to hepatic steatosis. Nat Med 2017;23:12981308. [PubMed: 29035368]

[45]. Assimakopoulos SF, Tsamandas AC, Louvros E, Vagianos CE, Nikolopoulou VN, Thomopoulos $\mathrm{KC}$, et al. Intestinal epithelial cell proliferation, apoptosis and expression of tight junction proteins in patients with obstructive jaundice. Eur J Clin Invest 2011;41:117-125. [PubMed: 20840373] 
[46]. Yan AW, Fouts DE, Brandl J, Starkel P, Torralba M, Schott E, et al. Enteric Dysbiosis Associated with a Mouse Model of Alcoholic Liver Disease. Hepatology 2011;53:96-105. [PubMed: 21254165]

[47]. Collado MC, Derrien M, Isolauri E, de Vos WM, Salminen S. Intestinal integrity and Akkermansia muciniphila, a mucin-degrading member of the intestinal microbiota present in infants, adults, and the elderly. Appl Environ Microbiol 2007;73:7767-7770. [PubMed: 17933936]

[48]. Derrien M, Collado MC, Ben-Amor K, Salminen S, de Vos WM. The Mucin degrader Akkermansia muciniphila is an abundant resident of the human intestinal tract. Appl Environ Microbiol 2008;74:1646-1648. [PubMed: 18083887]

[49]. Everard A, Belzer C, Geurts L, Ouwerkerk JP, Druart C, Bindels LB, et al. Cross-talk between Akkermansia muciniphila and intestinal epithelium controls diet-induced obesity. Proc Natl Acad Sci U S A 2013;110:9066-9071. [PubMed: 23671105]

[50]. Chen P, Torralba M, Tan J, Embree M, Zengler K, Starkel P, et al. Supplementation of saturated long-chain fatty acids maintains intestinal eubiosis and reduces ethanol-induced liver injury in mice. Gastroenterology 2015;148:203-214 e216. [PubMed: 25239591]

[51]. Kelly CJ, Zheng L, Campbell EL, Saeedi B, Scholz CC, Bayless AJ, et al. Crosstalk between Microbiota-Derived Short-Chain Fatty Acids and Intestinal Epithelial HIF Augments Tissue Barrier Function. Cell Host Microbe 2015;17:662-671. [PubMed: 25865369]

[52]. Bull-Otterson L, Feng W, Kirpich I, Wang Y, Qin X, Liu Y, et al. Metagenomic analyses of alcohol induced pathogenic alterations in the intestinal microbiome and the effect of Lactobacillus rhamnosus GG treatment. PLoS One 2013;8:e53028. [PubMed: 23326376]

[53]. Zhao H, Zhao C, Dong Y, Zhang M, Wang Y, Li F, et al. Inhibition of miR122a by Lactobacillus rhamnosus GG culture supernatant increases intestinal occludin expression and protects mice from alcoholic liver disease. Toxicol Lett 2015;234:194-200. [PubMed: 25746479]

[54]. Hong M, Kim SW, Han SH, Kim DJ, Suk KT, Kim YS, et al. Probiotics (Lactobacillus rhamnosus R0011 and acidophilus R0052) reduce the expression of toll-like receptor 4 in mice with alcoholic liver disease. PLoS One 2015;10:e0117451. [PubMed: 25692549]

[55]. Nath B, Levin I, Csak T, Petrasek J, Mueller C, Kodys K, et al. Hepatocyte-specific hypoxiainducible factor-1alpha is a determinant of lipid accumulation and liver injury in alcohol-induced steatosis in mice. Hepatology 2011;53:1526-1537. [PubMed: 21520168]

[56]. Nishiyama Y, Goda N, Kanai M, Niwa D, Osanai K, Yamamoto Y, et al. HIF-1alpha induction suppresses excessive lipid accumulation in alcoholic fatty liver in mice. J Hepatol 2012;56:441447. [PubMed: 21896344]

[57]. Forsyth CB, Voigt RM, Shaikh M, Tang Y, Cederbaum AI, Turek FW, et al. Role for intestinal CYP2E1 in alcohol-induced circadian gene-mediated intestinal hyperpermeability. Am J Physiol Gastrointest Liver Physiol 2013;305:G185-195. [PubMed: 23660503] 


\section{Highlights}

- Intestinal epithelial HIF-1a regulates multiple gut barrier protecting factors in response to alcohol exposure.

- Deletion of intestinal epithelial HIF-1a exacerbated gut dysbiosis, increased gut leakiness and bacterial product translocation leading to exacerbated ALD.

- $\quad$ Upregulation of intestinal HIF-1a may be a novel approach for ALD prevention/treatment. 


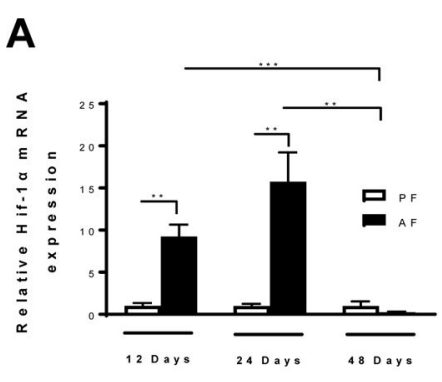

C

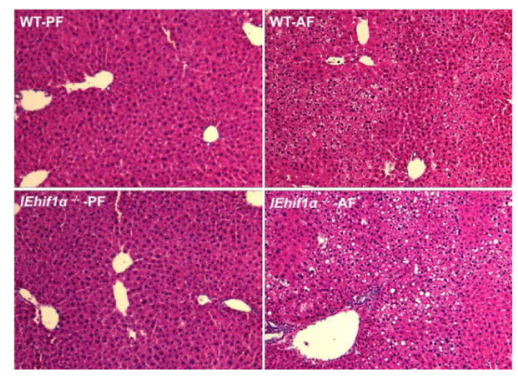

\section{E}

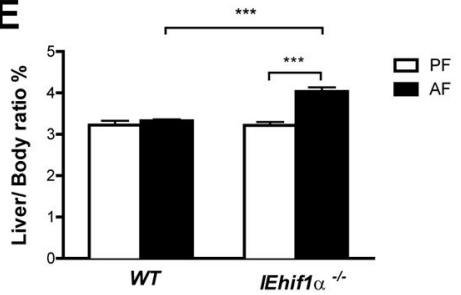

G

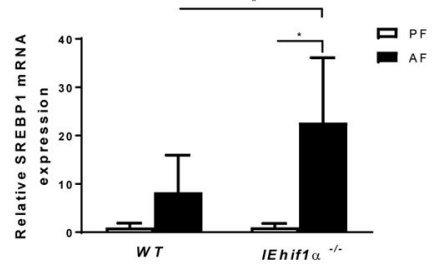

B

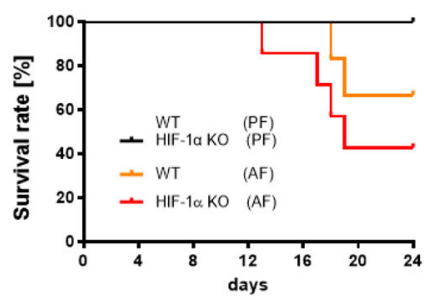

D

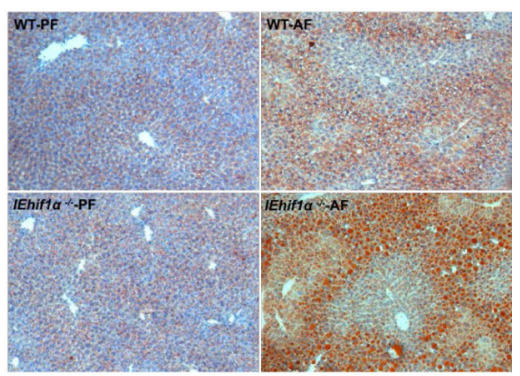

$\mathbf{F}$

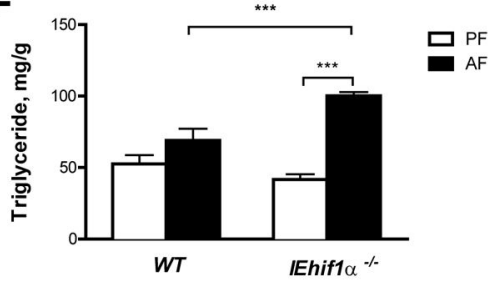

Figure 1.

Intestinal Hif1a KO (IEhifla ${ }^{-/-}$) mice display exacerbated chronic alcoholic liver injury and steatosis. WT and IEhifl $1 a^{-/}$mice were fed alcohol-containing Lieber DeCarli diet as described in the Material and Methods. (A) Hif-1a gene expression in WT mice fed 5\% alcohol-containing Lieber DeCarli diet for 12 ( $\mathrm{n}=4$ for PF group; $\mathrm{n}=6$ for AF group), 24 ( $\mathrm{n}=4$ for PF group; $\mathrm{n}=5$ for $\mathrm{AF}$ group), and 48 ( $\mathrm{n}=4$ for $\mathrm{PF}$ group; $\mathrm{n}=5$ for $\mathrm{AF}$ group) days, respectively. (B) Comparison of survival rate between WT and KO mice groups with or without $\mathrm{EtOH}$ ( $\mathrm{n}=9$ for each group). (C) Hematoxylin and eosin staining of the liver sections of the mice after PF or AF treatment for 24 days. Original magnification, $\times 10$. (D) Oil red O staining of the liver neutral lipids in mice described as in C. (E) Liver weight to body weight ratio of mice described as in C. (F) Quantification of hepatic triglyceride content in mice described as in C. (G) Gene expression of SREBP-1 in liver tissues of mice described as in C. Data are expressed as mean \pm SEM. AF, alcohol-fed; PF, pair-fed. 
A
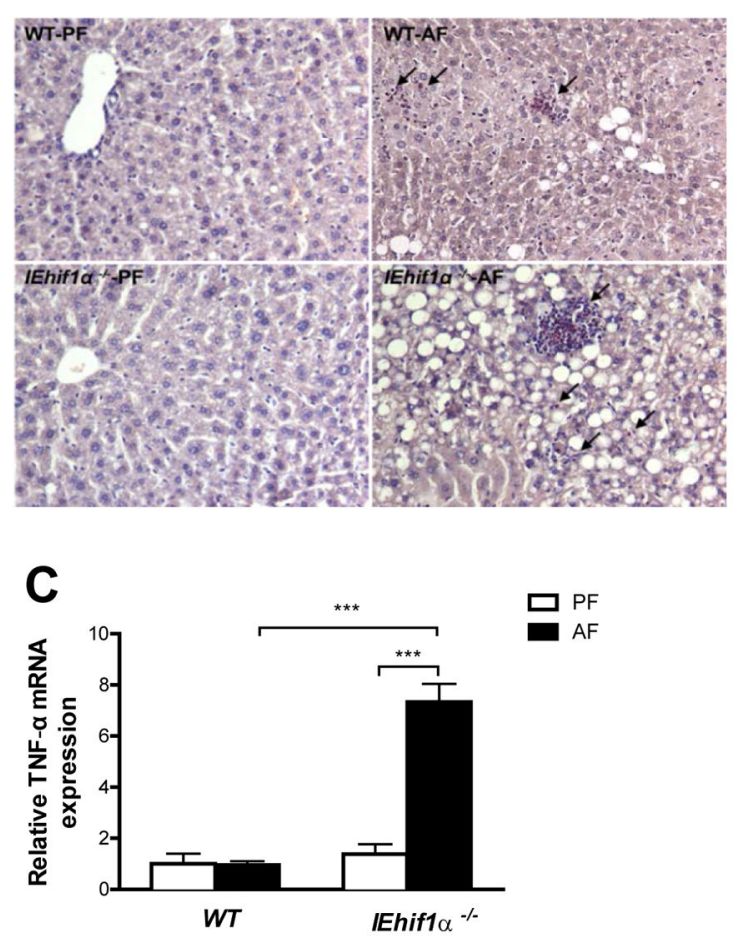

$\mathbf{E}$

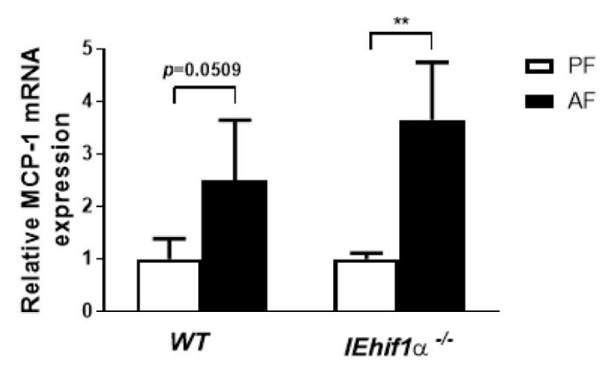

B
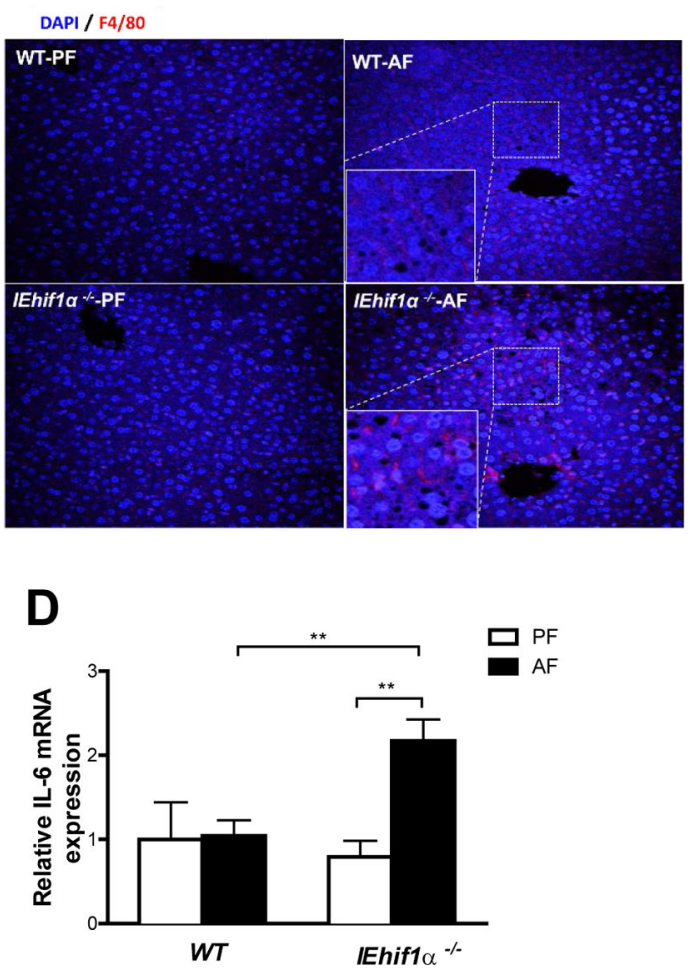

$\mathbf{F}$

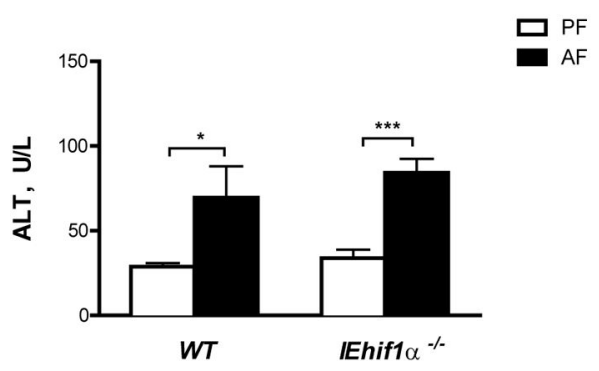

Figure 2.

Inflammatory responses in EtOH-induced liver injury. WT and IEhifla ${ }^{-/-}$mice were pairor alcohol-fed for 24 days. (A) Chloroacetate esterase (CAE) localization (cell-localized fuchsia staining) in livers. Original magnification, $\times 10$. Arrows indicate neutrophil infiltration. (B) F4/80 staining of the liver tissues. DAPI (blue) was used to counterstain nuclei. Original magnification, $\times 10$. (C, D, E) Hepatic gene expression of proinflammatory cytokines TNF-a, IL-6 and chemokine MCP-1, respectively. (F) Serum alanine aminotransferase (ALT) activities. Data are expressed as mean \pm SEM. AF, alcohol-fed; PF, pair-fed. 
A

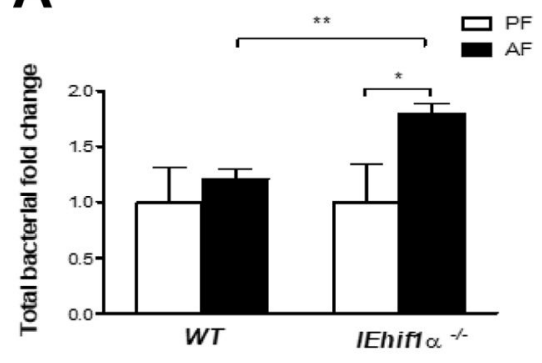

C
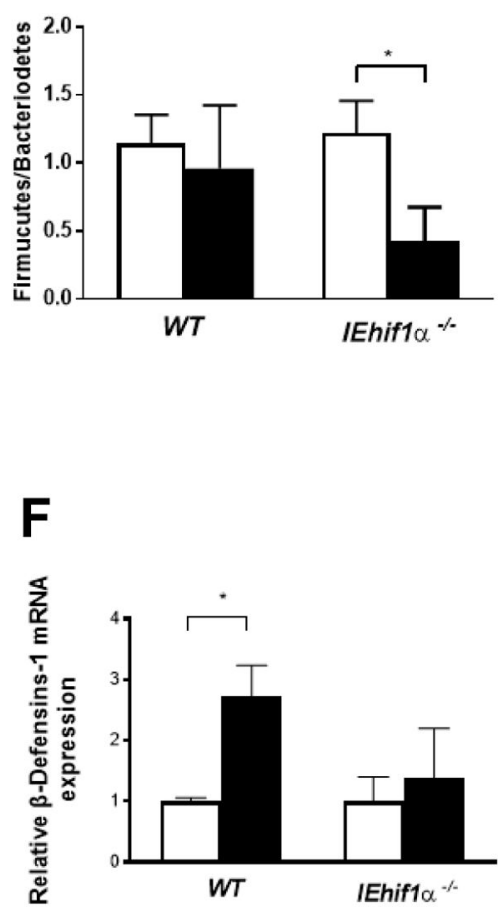

B

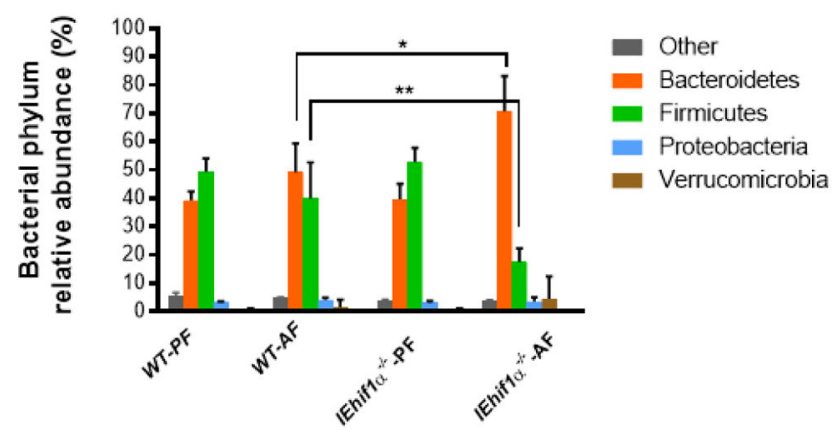

D

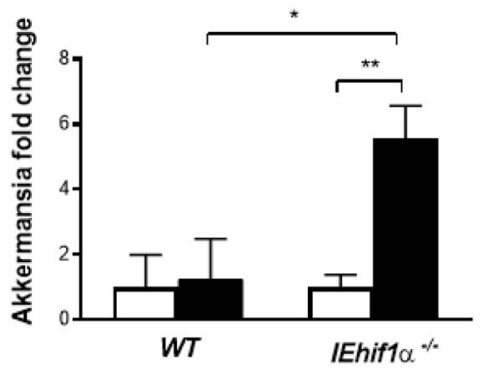

G

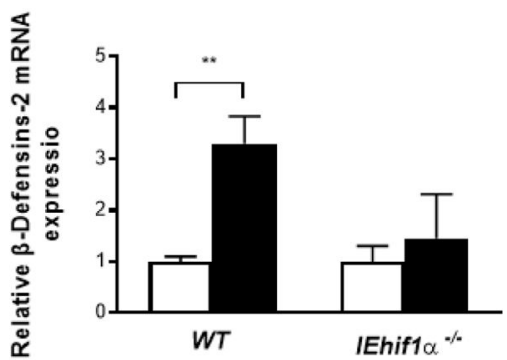

$\mathbf{E}$

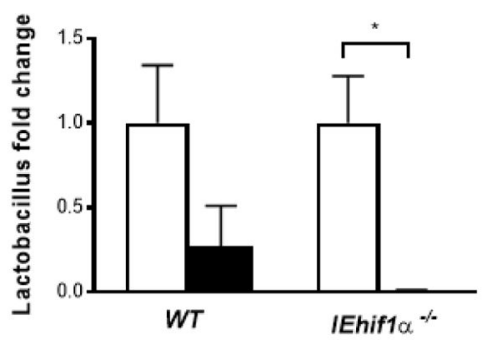

Figure 3.

Effect of Hifla on alcohol-induced dysbiosis. Mice were treated as described in Fig 2. (A) The total bacterial load in the cecum of mice. (B) Phylum levels of microbiota in fecal samples. (C) The ratio of Firmucutes/bacteriodetes level. (D, E) Akkermansia (Verrucomicrobia phylum) and Lactobacillus (Firmicutes phylum) levels. (F, G, H) $\beta$ Defensins ( $\beta$-Defensins-1,2) and cathelicidin-related antimicrobial peptide (CRAMP) gene expression in the ileal tissues. Data are expressed as mean \pm SEM. AF, alcohol-fed; PF, pairfed. 


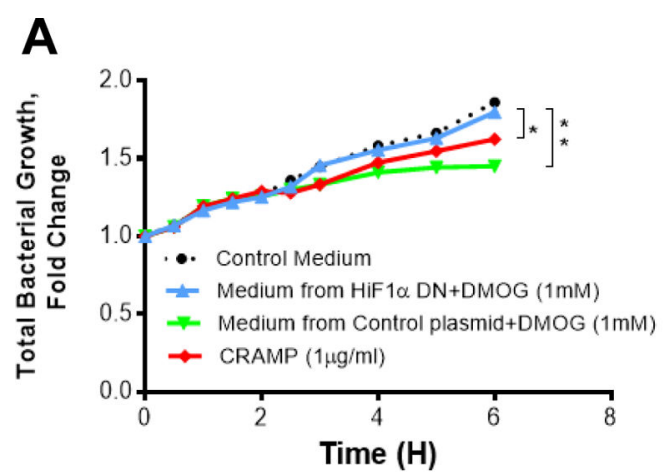

C

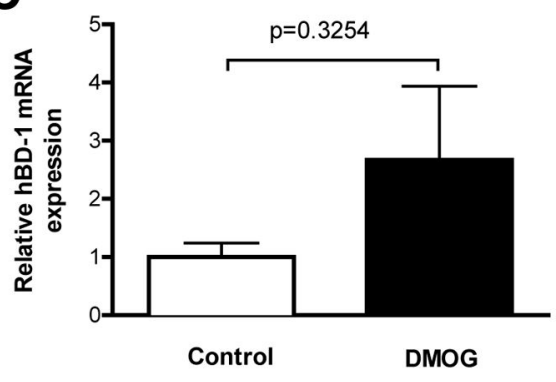

E

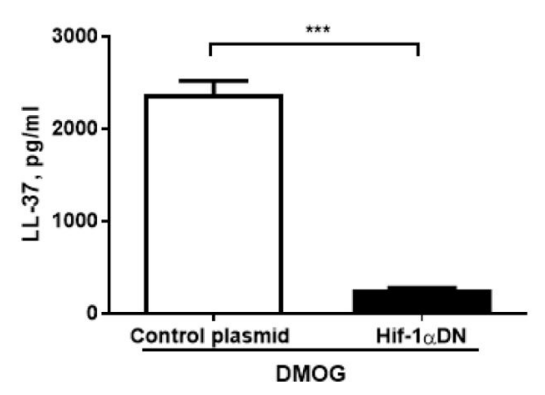

B

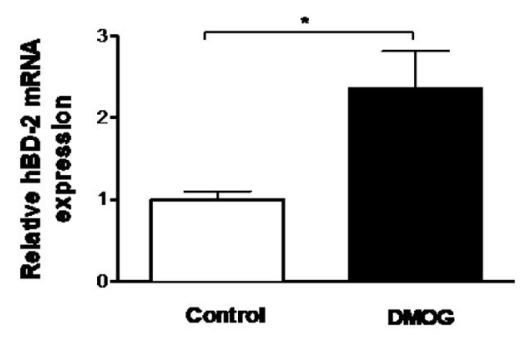

D

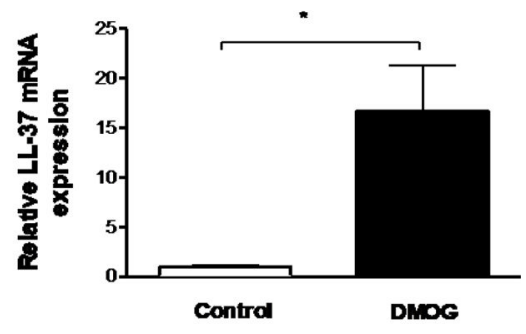

Figure 4.

Effect of HIF activation in T84 cells on the bacterial growth and antimicrobial peptide gene expression. (A) Mouse fecal bacterial growth in response to control plasmid+DMOG,

HIF-1aDN+DMOG, and synthetic CRAMP peptide. (B-D) Gene expression of LL-37 and human $\beta$-Defensins (hBD-1, 2). (E) The medium concentration of LL-37 by ELISA. Data are expressed as mean \pm SEM of a minimum of three independent experiments. 
A
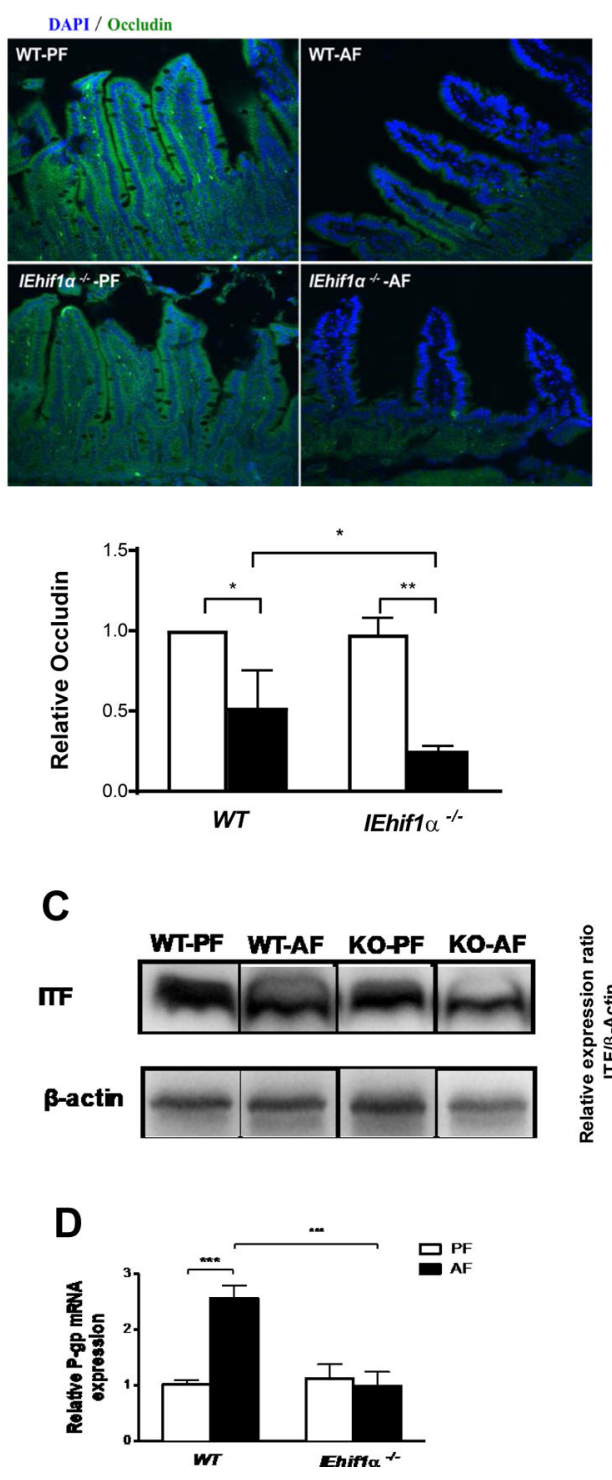

B

DAPI / Claudin-1
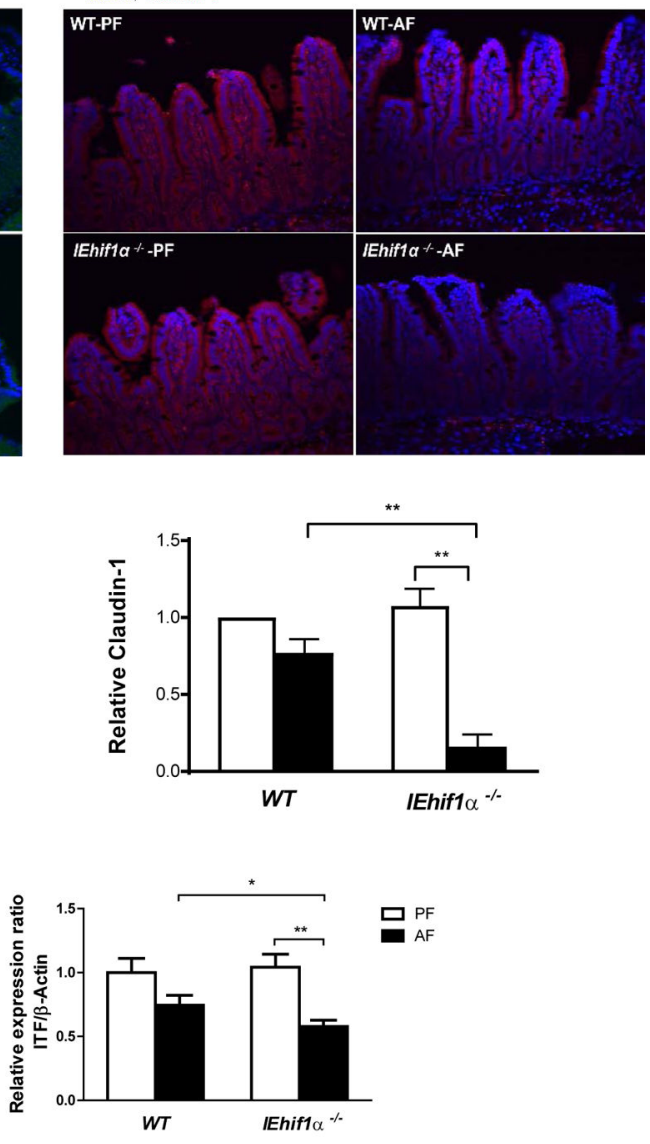

Figure 5.

Effect of Hif-1a on intestinal barrier integrity. Mice were treated as described in Fig 2. (A, B) Immunoflorescent staining of occludin (green) and claudin-1 (blue) in the ileal tissues. DAPI (blue) was used to counterstain of nuclei. Original magnification, $\times 10$. (C) ITF protein Western blotting and quantification of ileal tissues normalized by $\beta$-actin. The bands are composite images of selected bands; and that the corresponding $\beta$-actin controls are shown. (D) P-gp gene expression in ileal tissues. Data are expressed as mean \pm SEM. (AF, alcoholfed; PF, pair-fed). 
A

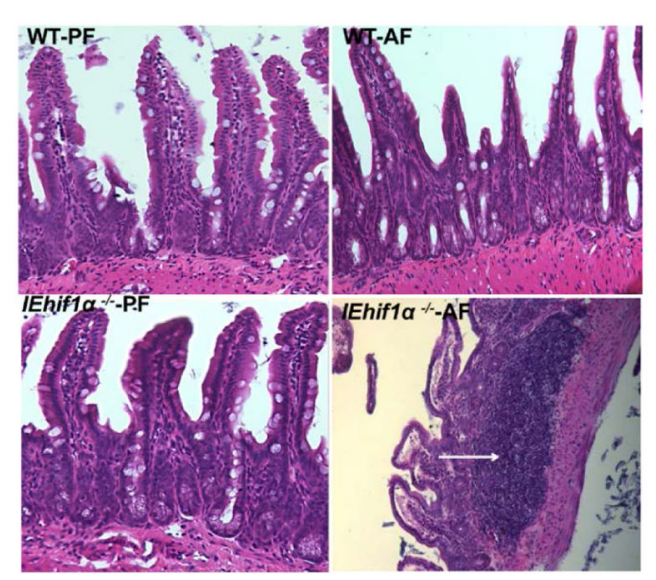

C

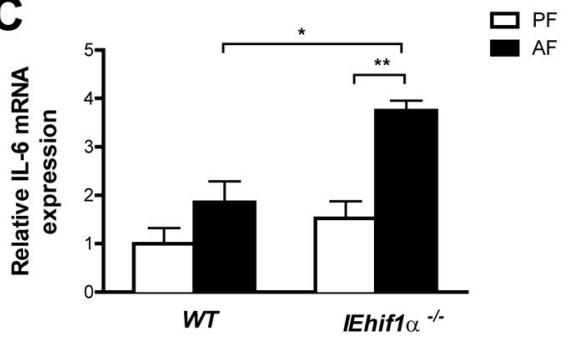

$\mathbf{E}$

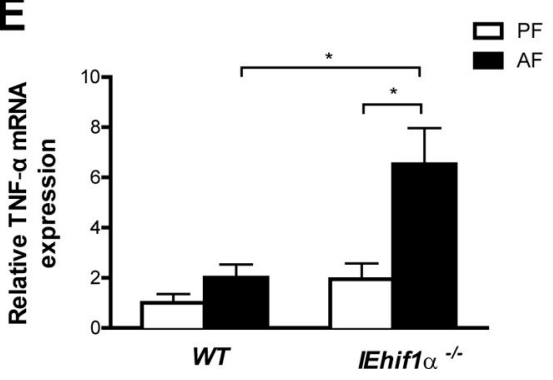

B
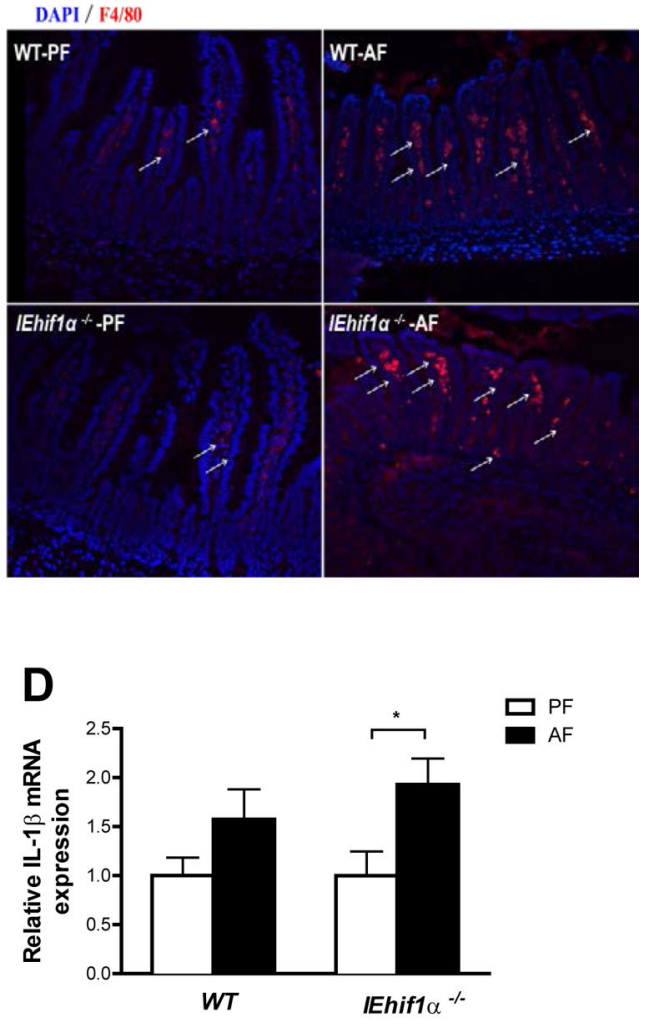

Figure 6.

Effect of Hif-1a on intestinal inflammation. Mice were treated as described in in Fig 2. (A) HE staining of ileal tissues. Original magnification, $\times 10$. (B) Immunofluorescent staining of F4/80 (red) showed increased macrophages in ileum of AF IEhifla ${ }^{-/}$mice (arrows). DAPI (blue) was used to visualize nuclei. Original magnification: $\times 10$. (C, D, E) Ileum IL-6, IL-1 $\beta$ and TNF- $a$ mRNA levels. Data are expressed as mean \pm SEM. AF, alcohol-fed; PF, pair-fed. 
A
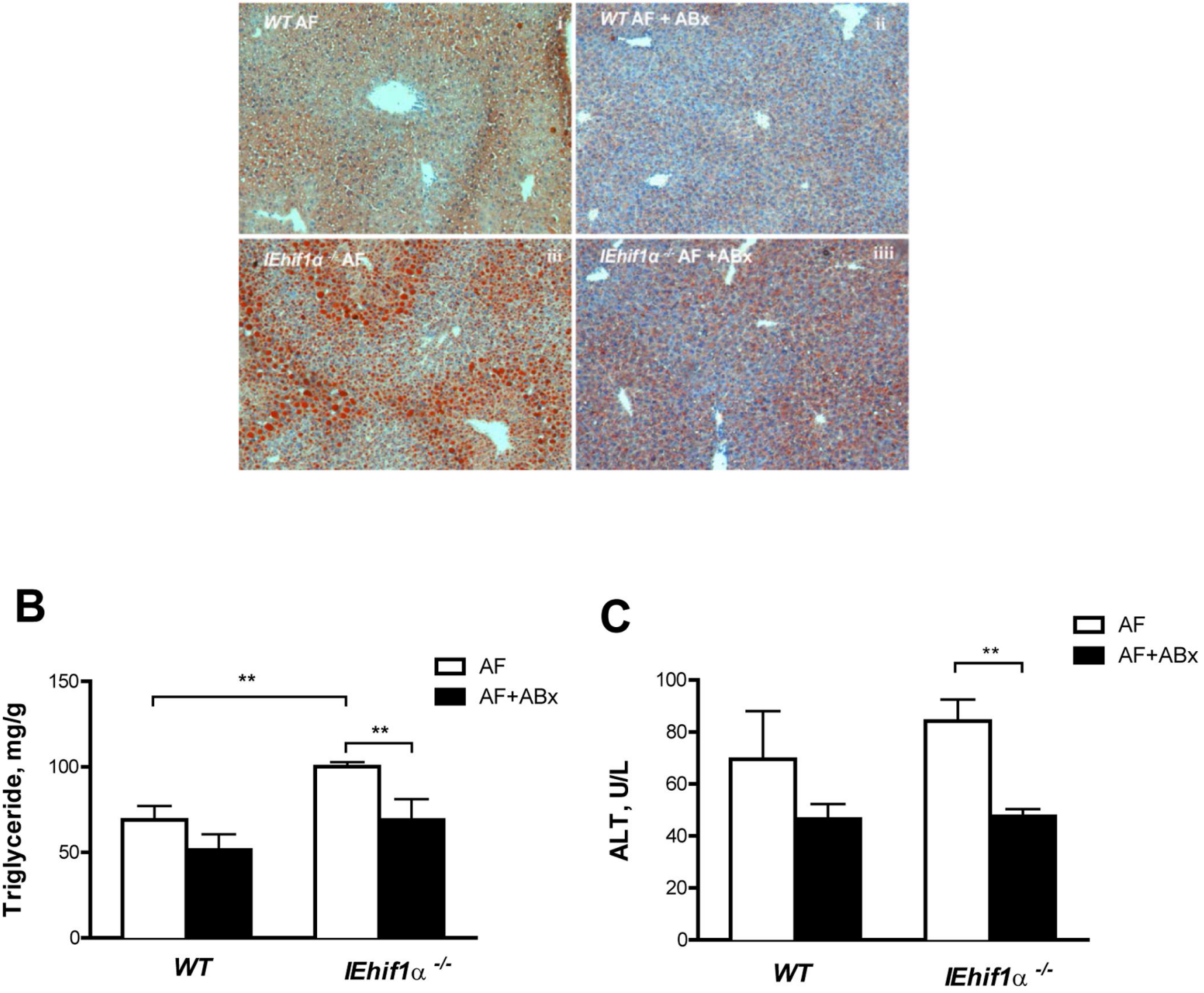

Figure 7.

Effects of non-absorbable broad-spectrum antibiotics ( $\mathrm{ABx})$ on EtOH-induced liver steatosis and injury. WT and IEhifl $1 a^{-/}$mice were fed 5\% alcohol liquid diets for 24 days (n=4-6). Antibiotics treatment was started at day 12 after alcohol feeding, and mice were gavaged daily until harvesting. (A) Oil red O staining of hepatic tissues. Original magnification, $\times 10$. (B) Quantification of hepatic triglyceride content. (C) Serum ALT levels. Data are mean \pm SEM. AF, alcohol-fed; PF, pair-fed. 
A

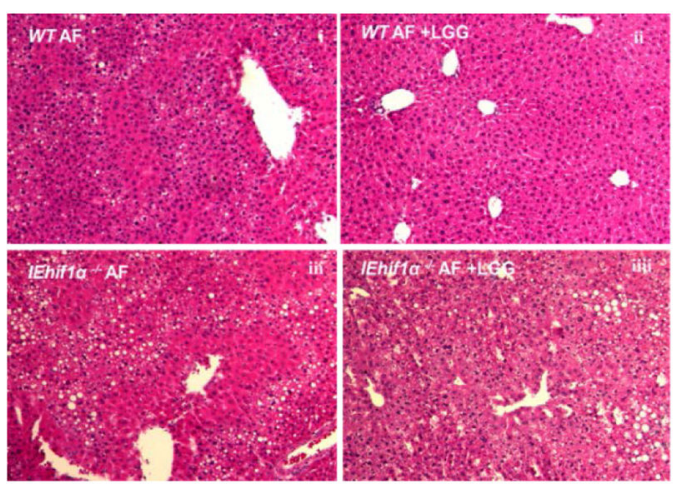

C

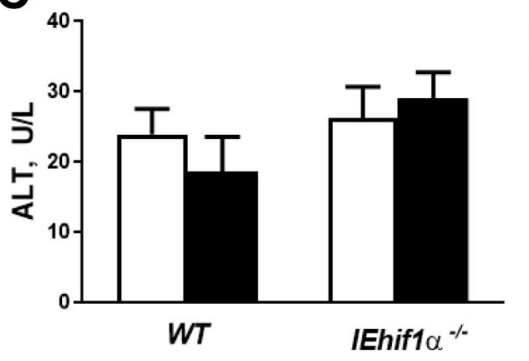

E

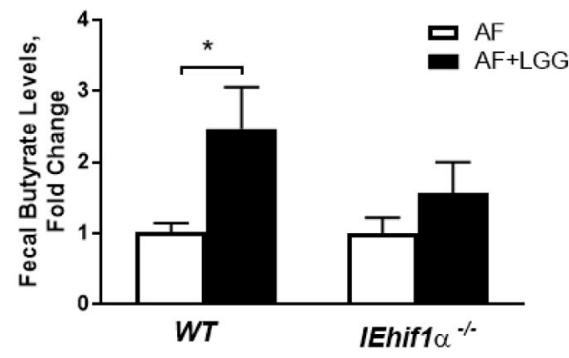

B

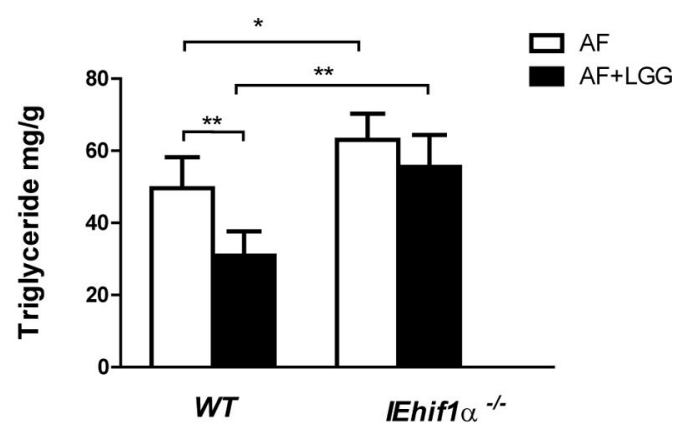

D

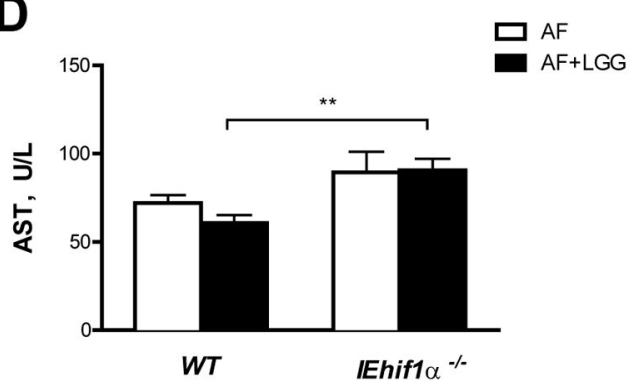

$\mathbf{F}$

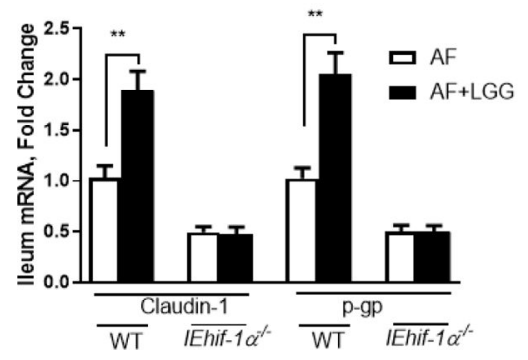

Figure 8.

Effects of Hif-1a on Lactobacillus rhamnosus GG treatment in ALD. Mice were fed alcohol following the NIAAA model ( $\mathrm{n}=5$ for each group). (A) HE staining revealed LGG administration reduced steatosis by alcohol exposure in WT mice. However, LGG protective effects were diminished in IEhifla $a^{-/}$mice. (B) Quantification of hepatic triglyceride content. (C,D) Serum ALT and AST levels. (E) Fecal butyrate concentration. (F) Ileum Claudin-1 and P-gp mRNA levels. Data are expressed as mean \pm SEM. AF, alcohol-fed; PF, pair-fed. 